Article

\title{
Dietary Fiber Analysis of Four Pulses Using AOAC 2011.25: Implications for Human Health
}

\author{
Yiran Chen ${ }^{1}$, Rebecca McGee ${ }^{2}$, George Vandemark ${ }^{2}$, Mark Brick ${ }^{3}$ and Henry J. Thompson ${ }^{1, *}$ \\ 1 Cancer Prevention Laboratory, Colorado State University, Fort Collins, CO 80523, USA; \\ yiran.chen@colostate.edu \\ 2 Agricultural Research Service, United States Department of Agriculture, Washington State University, \\ Pullman, WA 99164, USA; rebecca.mcgee@ars.usda.gov (R.M.); george.vandemark@ars.usda.gov (G.V.) \\ 3 Department of Soil and Crop Sciences, Colorado State University, Fort Collins, CO 80523, USA; \\ mark.brick@colostate.edu \\ * Correspondence: henry.thompson@colostate.edu; Tel.: +1-970-491-7748; Fax: +1-970-491-3542
}

Received: 11 November 2016; Accepted: 16 December 2016; Published: 21 December 2016

\begin{abstract}
Chickpeas, common beans, dry peas, and lentils are pulse crops that have been a cornerstone of the human diet since the inception of agriculture. However, the displacement of pulses from the diet by low fiber protein alternatives has resulted in a pervasive deficiency referred to as the dietary fiber gap. Using an analytical method American Association of Analytical Chemists (AOAC) 2011.25 that conforms to the Codex Alimentarius Commission consensus definition for dietary fiber, the fiber content of these pulse crops was evaluated in seed types used for commercial production. These pulse crops have 2 to 3 times more fiber per $100 \mathrm{~g}$ edible portion than other dietary staples. Moreover, there is marked variation in fiber content among cultivars of the same crop. We conclude that pulse crop consumption should be emphasized in efforts to close the dietary fiber gap. The substantial differences in fiber content among currently available cultivars within a crop can be used to further improve gains in fiber intake without the need to change dietary habits. This provides a rationale for cultivar-based food labeling.
\end{abstract}

Keywords: dietary fiber; pulse crops; dietary fiber gap; common bean; chickpeas; dry peas; lentils; AOAC 2011.25

\section{Introduction}

Dietary fiber is an important non-nutritive component of food, and is believed to have various benefits to human health [1]. In many countries, such as the United States and Canada, the intake of dietary fiber is $50 \%$ to $70 \%$ below recommended levels in greater than $95 \%$ of the population $[2,3]$. We recently advanced the concept that low dietary fiber intake could be partially accounted for by the infrequent or low consumption of pulse crops [4]. As defined by Food and Agriculture Organization (FAO) of the United Nations, pulses are grain legumes; the four most widely consumed pulse crops globally are chickpeas (Cicer arietinum L.), common beans (Phaseolus vulgaris L.), dry peas (Pisum sativum L.), and lentils (Lens culinaris L.) [5]. In many cultures, these pulse crops are food staples; i.e. they are eaten daily in large amounts and are both affordable and accessible to the population [6]. While this continues to be true in many developing countries, in other countries, such as the United States and Canada, pulse consumption has been displaced with animal products, oil seed legumes such as soy (protein isolate), and peanuts. Consequently, while the FAO recommends the consumption of cereal grains to pulses in a ratio of 2:1 (food servings), the current pattern of consumption globally is 8:1 [7]. Interestingly, it has been shown in both the United States and Canada that individuals whose consumption of pulses is high (highest quartile of dietary intake) meet recommended levels of dietary fiber intake in comparison to non-pulse consumers who eat the same daily number of servings of cereal grains $[8,9]$. 
While the first use of the term dietary fiber is attributed to Hipsley in 1953 [10], the hypothesis that dietary fiber is associated with human health benefits is attributed to the research team of Burkitt, Painter, Walker, and Trowel [11]. To support the conceptual development of their ideas, Trowel published a definition for dietary fiber in 1976 that was used for method development and policy formulation for over 20 years [12]. With the expansion of scientific knowledge, efforts to update Trowel's definition have been ongoing [13]. Those efforts recently culminated in an international consensus by the authority responsible for determining global food standards and guidelines (the Codex Alimentarius Commission), with the adoption of a new definition of dietary fiber in 2009 by the Codex Committee on Nutrition and Foods for Special Dietary Uses [14]. The definition states that: "Dietary fiber consists of carbohydrate polymers with 10 or more monomeric units, which are not hydrolyzed by the endogenous enzymes in the small intestine of humans". This definition resulted in a review of available analytical methods and whether the existing methods could define the fiber components included in the new definition. An integrated total dietary fiber analytical method was developed by McCleary et al. to comply with the 2009 CODEX definition and accurately measure total dietary fiber (TDF) as calculated by insoluble dietary fiber (IDF), soluble dietary fiber (SDF), and non-digestible oligosaccharides (OLIGO) content. This integrated method was subjected to inter-laboratory evaluations and initially accepted as AOAC 2009.01 [15]. Additional inter-laboratory evaluations resulted in the acceptance of AOAC method 2011.25 [16].

The consensus definition of dietary fiber and the acceptance of a method by which it can be reliably determined resolved a long-standing barrier to the field of dietary fiber and human health. We now apply AOAC 2011.25 as reported in [17] to the comparative analysis of pulse crops, since this information is not available in current nutrient databases. We also report on variability in dietary fiber among commercially-grown cultivars of each crop and demonstrate why efforts to brand foods with varietal information could help the consumer make more informed choices while providing an opportunity for food producers to advertise this value-added aspect of their product without the need to make any health claims.

\section{Materials and Methods}

\subsection{Experimental Design}

Chickpea, common bean, dry pea, and lentil seed were selected by plant breeders familiar with commercial cultivars of each pulse crop grown in the United States. Chickpea was selected by Geroge Vandemark, common bean by Mark Brick, and dry peas and lentils were selected by Rebecca McGee. Seed was harvested from two locations, and within each location, two samples (replicates) of the same cultivar were obtained from different field plots.

\subsection{Sample Preparation}

Seed samples for analysis were treated as follows. Approximately $1 \mathrm{~g}$ of each seed sample was ground into a fine powder using a mechanical grinder (KRUPS Tipo203, Krups-USA, Peoria, IL, USA). Two subsamples of each sample were weighed and transferred into a 50-mL plastic Falcon tube (Corning Science Mexico, Reynosa, Mexico), and then $8 \mathrm{~mL}$ of Milli-Q water was added. The sample was vortexed with Baxter S/P vortex mixer (Baxter Diagnosis Inc., Deerfield, IL, USA) until the powder was well dispersed in water. The tubes were then placed in an autoclave, where they were autoclaved for $65 \mathrm{~min}$ at $115^{\circ} \mathrm{C}$ with pressure of $76 \mathrm{kPa}$ to simulate the cooking process. After cooking, the sample was cooled to room temperature and the contents of the tubes were fully homogenized using a Polytron ${ }^{\circledR}$ PT10/35 (Kinematica, Lucerne, Switzerland) with an $S$ type probe for approximately $15 \mathrm{~s}$ on speed 6. The probe was washed twice with $6 \mathrm{~mL}$ of Milli-Q water (EMD Millipore, Darmstadt, Germany) in a VWR Culture tube (VWR Scientific, West Chester, PA, USA) $17 \times 100 \mathrm{~mm}$ after homogenization to remove all seed particles. The liquids from the two washings were transferred to the original $50 \mathrm{~mL}$ conical tube containing the homogenized seed sample. The final sample volume was approximately $20 \mathrm{~mL}$. The conical tubes with the homogenized seed sample were stored at $-80^{\circ} \mathrm{C}$ until analysis. 


\subsection{AOAC 2011.25 Integrated Total Dietary Fiber Assay}

Total integrated dietary fiber content was quantified per the AOAC 2011.25 method as modified by Kleintops et al. (2011) using a commercial assay kit (K-INTDF) purchased from Megazyme International, Wicklow, Ireland. The assay was carried out with modifications, as published in detail by our laboratory [17]. Briefly, the analysis of insoluble and soluble dietary fiber was per AOAC2011.25 without modification. Since oligosaccharides in pulses are limited to galactans, we used High Performance Liquid Chromatography (HPLC) separation with electrochemical detection to improve the sensitivity and specificity of the assay for raffinose, stachyose, and verbascose.

\subsection{Statistical Analysis}

An analysis of variance was carried out to compare entry means on all variables using the Procedure for General Linear Models (Proc GLM ) in SAS version 9.2 (SAS Institute Inc., Cary, NC, USA). Tukey's multiple mean comparison method $(p<0.05)$ was used to determine significance among entry means and among crops for all variables.

\section{Results}

\subsection{Chickpea}

Twenty-four kabuli chickpea entries were evaluated; they included five varieties and 19 breeding lines from the USDA-ARS Grain Legume Genetics and Physiology Research Unit (Pullman, WA, USA). Seed was obtained from field locations in Genesee, ID, and Pullman, WA. Selection of chickpeas was limited to the kabuli type, since that is the predominant type grown in the North America. Within each location, seed was collected from two different field plots. Since the goal of this study was to determine concentrations of dietary fiber components and total dietary fiber in cultivars of chickpea as they would be purchased and consumed, the four independent values for each variety were averaged and are reported in Table 1.

Table 1. Dietary fiber content of chickpea cultivars and breeding lines.

\begin{tabular}{|c|c|c|c|c|}
\hline Entry & $\operatorname{IDF}^{\mathrm{a}, \mathrm{b}}$ & $\operatorname{SDF}^{a, b}$ & OLIGO $^{a, b}$ & TDF $^{a, b}$ \\
\hline Billy beans & $15.4 \pm 1.9 \mathrm{ABCD}$ & $3.2 \pm 0.3^{\mathrm{EFG}}$ & $3.2 \pm 0.1 \mathrm{AB}$ & $21.8 \pm 1.8^{\text {BCDEFG }}$ \\
\hline CA04900843C & $16.9 \pm 0.9^{\mathrm{A}}$ & $3.4 \pm 0.4^{\mathrm{DEF}}$ & $3.1 \pm 0.2^{\mathrm{ABC}}$ & $23.4 \pm 1.0 \mathrm{ABC}$ \\
\hline CA0690B0427C & $15.1 \pm 1.2 \mathrm{ABCD}$ & $5.8 \pm 0.2^{\mathrm{A}}$ & $1.9 \pm 0.9 \mathrm{GH}$ & $22.8 \pm 1.7 \mathrm{ABCDEF}$ \\
\hline CA0690B250C & $16.7 \pm 0.7 \mathrm{AB}$ & $3.3 \pm 0.2$ DEFG & $3.1 \pm 0.1 \mathrm{ABC}$ & $23.2 \pm 0.6 \mathrm{ABCD}$ \\
\hline СА0790B0034С & $16.5 \pm 0.9 \mathrm{ABCD}$ & $5.7 \pm 0.6^{\mathrm{A}}$ & $2.7 \pm 0.6^{\mathrm{ABCDEF}}$ & $24.9 \pm 1.1^{\mathrm{A}}$ \\
\hline CA0790B0042C & $15.9 \pm 1.0 \mathrm{ABCD}$ & $5.8 \pm 0.6^{\mathrm{A}}$ & $2.8 \pm 0.4 \mathrm{ABCDE}$ & $24.5 \pm 1.0^{\mathrm{A}}$ \\
\hline CA0790B0043C & $15.6 \pm 1.1 \mathrm{ABCD}$ & $2.0 \pm 0.1^{\mathrm{I}}$ & $3.3 \pm 0.2 \mathrm{AB}$ & $21.0 \pm 1.2$ DEFG \\
\hline CA0790B0053C & $15.0 \pm 2.6^{\mathrm{ABCD}}$ & $2.0 \pm 0.1^{\mathrm{I}}$ & $3.0 \pm 0.1 \mathrm{ABC}$ & $19.9 \pm 2.7^{\mathrm{G}}$ \\
\hline СА0790B0054C & $15.6 \pm 1.2 \mathrm{ABCD}$ & $2.4 \pm 0.2^{\mathrm{HI}}$ & $2.9 \pm 0.2 \mathrm{ABCD}$ & $20.9 \pm 1.4$ DEFG \\
\hline CA0790B0547C & $15.6 \pm 0.6^{\mathrm{ABCD}}$ & $3.2 \pm 0.3^{\mathrm{EFG}}$ & $2.1 \pm 0.7$ DEFGH & $20.9 \pm 0.7 \mathrm{EFG}$ \\
\hline CA0790B0549C & $15.8 \pm 1.9 \mathrm{ABCD}$ & $3.2 \pm 0.6^{\mathrm{EFG}}$ & $2.5 \pm 0.9$ BCDEFG & $21.4 \pm 2.7$ CDEFG \\
\hline CA0790B0642C & $16.7 \pm 1.1 \mathrm{AB}$ & $2.8 \pm 0.3^{\mathrm{GH}}$ & $2.1 \pm 0.6^{\mathrm{EFGH}}$ & $21.5 \pm 0.9$ CDEFG \\
\hline CA0790B0733C & $15.0 \pm 1.2 \mathrm{ABCD}$ & $3.0 \pm 0.5^{\mathrm{EFG}}$ & $1.8 \pm 1.0 \mathrm{GH}$ & $19.8 \pm 2.0^{\mathrm{G}}$ \\
\hline CA0890B0085W & $14.4 \pm 1.3^{\mathrm{D}}$ & $3.3 \pm 0.3 \mathrm{DEF}$ & $1.9 \pm 0.2 \mathrm{FGH}$ & $19.6 \pm 1.4^{\mathrm{G}}$ \\
\hline CA0890B0429C & $15.9 \pm 0.9 \mathrm{ABCD}$ & $2.9 \pm 0.4^{\mathrm{FGH}}$ & $2.1 \pm 1.0$ DEFGH & $20.9 \pm 1.8^{\text {DEFG }}$ \\
\hline CA0890B0434C & $16.0 \pm 1.0 \mathrm{ABCD}$ & $3.0 \pm 0.6^{\mathrm{FG}}$ & $2.4 \pm 0.3$ CDEFGH & $21.3 \pm 1.2$ CDEFG \\
\hline CA0890B0496C & $14.7 \pm 1.2^{\mathrm{BCD}}$ & $3.0 \pm 0.3^{\mathrm{FG}}$ & $1.9 \pm 1.1$ FGH & $19.5 \pm 2.2^{G}$ \\
\hline CA0890B0531C & $16.2 \pm 3.8 \mathrm{ABCD}$ & $2.9 \pm 0.2 \mathrm{FG}$ & $1.6 \pm 0.8^{\mathrm{HI}}$ & $20.7 \pm 3.4^{\mathrm{FG}}$ \\
\hline CA0890B0551C & $15.5 \pm 1.6^{\mathrm{ABCD}}$ & $3.8 \pm 0.2 \mathrm{CD}$ & $1.0 \pm 0.9^{\mathrm{I}}$ & $20.3 \pm 1.9^{\mathrm{G}}$ \\
\hline CA0890B0628W & $14.6 \pm 1.4 \mathrm{CD}$ & $4.4 \pm 0.5^{\mathrm{B}}$ & $2.1 \pm 1.2$ DEFGH & $21.1 \pm 2.0$ DEFG \\
\hline CA0890B0648W & $15.7 \pm 1.2 \mathrm{ABCD}$ & $4.3 \pm 0.4^{\mathrm{BC}}$ & $2.8 \pm 0.1 \mathrm{ABCDE}$ & $22.8 \pm 1.4 \mathrm{ABCDEF}$ \\
\hline CDC-Frontier & $17.1 \pm 1.9^{\mathrm{A}}$ & $3.6 \pm 0.2^{\mathrm{DE}}$ & $3.2 \pm 0.1 \mathrm{AB}$ & $23.8 \pm 1.9 \mathrm{AB}$ \\
\hline Sawyer & $16.6 \pm 1.4 \mathrm{ABC}$ & $3.4 \pm 0.3^{\mathrm{DEF}}$ & $3.4 \pm 0.1 \mathrm{~A}$ & $23.4 \pm 1.4 \mathrm{ABC}$ \\
\hline Sierra & $16.1 \pm 1.7 \mathrm{ABCD}$ & $3.5 \pm 0.1^{\mathrm{DEF}}$ & $3.5 \pm 0.2^{\mathrm{A}}$ & $23.1 \pm 1.5 \mathrm{ABCDE}$ \\
\hline Overall Mean & $15.8 \pm 1.5$ & $3.5 \pm 1.5$ & $2.5 \pm 0.9$ & $21.8 \pm 2.2$ \\
\hline$p$-value & 0.498 & $<0.0001$ & $<0.0001$ & $<0.0001$ \\
\hline
\end{tabular}

${ }^{a}$ Values are expressed as percent of dry weight; means \pm SD; ${ }^{b}$ Means followed by different superscripted letters within a column are significantly different $(p<0.05)$ according to Tukey's mean separation test. Abbreviations: IDF: insoluble dietary fiber; SDF: soluble dietary fiber; OLIGO: total oligosaccharides (raffinose + stachyose + verbascose); TDF: total dietary fiber (IDF + SDF + OLIGO). 
The average total dietary fiber for all chickpea cultivars was $21.8 \%$, with a range of $19.5 \%$ to $24.9 \%$. Average insoluble dietary fiber across the 24 cultivars was $15.8 \%$, while the range was $14.4 \%$ to $17.1 \%$. The average soluble dietary fiber for all cultivars was 3.5\%, with a range of $2.0 \%$ to $5.8 \%$. The average for total oligosaccharides was $2.5 \%$, with a range between $1.0 \%$ and $3.5 \%$.

\subsection{Common Bean}

Twenty-six common bean entries were evaluated. Selection of seed included cultivars from market classes commonly grown in North America. Beans were obtained from two locations (Colorado and North Dakota), and within each location, seed was collected from two different field plots. Since the goal of this study was to determine concentrations of dietary fiber components and total dietary fiber in commercial cultivars of common bean as they would be purchased by a consumer, the four independent values for each variety were averaged and are reported in Table 2.

Table 2. Dietary fiber content of common bean cultivars.

\begin{tabular}{|c|c|c|c|c|}
\hline Variety & $\operatorname{IDF}^{a, b}$ & $\operatorname{SDF}^{a, b}$ & OLIGO $^{a, b}$ & $\mathrm{TDF}^{\mathrm{a}, \mathrm{b}}$ \\
\hline Agassiz & $13.1 \pm 0.5^{\mathrm{DEF}}$ & $7.0 \pm 0.2$ EFGHI & $4.1 \pm 0.2^{\mathrm{BCDE}}$ & $24.1 \pm 0.6^{\mathrm{E}}$ \\
\hline Arthur & $14.4 \pm 0.5^{\mathrm{ABCDE}}$ & $6.8 \pm 0.4$ FGHI & $3.6 \pm 0.2^{\mathrm{E}}$ & $24.8 \pm 0.5 \mathrm{CDE}$ \\
\hline Avalanche & $14.3 \pm 0.9 \mathrm{ABCDE}$ & $6.6 \pm 0.6^{\mathrm{HI}}$ & $3.9 \pm 0.4 \mathrm{CDE}$ & $24.8 \pm 0.9 \mathrm{DE}$ \\
\hline Sequoia & $13.4 \pm 0.7^{\mathrm{CDEF}}$ & $7.1 \pm 0.1 \mathrm{EFGH}$ & $4.3 \pm 0.4^{\mathrm{BCD}}$ & $24.9 \pm 0.6^{\mathrm{CDE}}$ \\
\hline Blackhawk & $13.2 \pm 0.3^{\mathrm{CDEF}}$ & $7.1 \pm 0.7 \mathrm{EFGH}$ & $4.7 \pm 0.2 \mathrm{AB}$ & $25.1 \pm 0.6^{\mathrm{BCDE}}$ \\
\hline Fisher & $13.8 \pm 0.9 \mathrm{ABCDEF}$ & $6.9 \pm 0.3^{\mathrm{EFGHI}}$ & $4.4 \pm 0.4^{\mathrm{BC}}$ & $25.2 \pm 0.4^{\mathrm{BCDE}}$ \\
\hline Buckskin & $13.2 \pm 0.7 \mathrm{DEF}$ & $7.8 \pm 0.5^{\mathrm{CDEFGH}}$ & $4.3 \pm 0.2^{\mathrm{BCD}}$ & $25.3 \pm 1.2^{\mathrm{BCDE}}$ \\
\hline Beryl & $12.3 \pm 0.4^{\mathrm{F}}$ & $9.2 \pm 0.3^{\mathrm{AB}}$ & $4.0 \pm 0.2^{\mathrm{CDE}}$ & $25.5 \pm 0.3^{\text {BCDE }}$ \\
\hline Harrowhawk & $13.8 \pm 0.6^{\mathrm{BCDEF}}$ & $7.6 \pm 0.3 \mathrm{CDEFGH}$ & $4.1 \pm 0.1 \mathrm{BCDE}$ & $25.5 \pm 0.5^{\mathrm{BCDE}}$ \\
\hline Hyden & $13.6 \pm 1.0^{B C D E F}$ & $8.1 \pm 0.5^{\text {BCDEFG }}$ & $3.9 \pm 0.2 \mathrm{CDE}$ & $25.6 \pm 1.1 \mathrm{ABCDE}$ \\
\hline Bill Z & $14.4 \pm 1.0^{\mathrm{ABCDE}}$ & $7.6 \pm 0.5^{\mathrm{CDEFGH}}$ & $3.6 \pm 0.3^{\mathrm{E}}$ & $25.6 \pm 1.5^{\mathrm{ABCDE}}$ \\
\hline Jaguar & $13.7 \pm 0.9^{\mathrm{BCDEF}}$ & $7.8 \pm 0.2 \mathrm{CDEFGH}$ & $4.2 \pm 0.2^{\mathrm{BCDE}}$ & $25.7 \pm 0.8 \mathrm{ABCDE}$ \\
\hline Bunsi & $14.1 \pm 1.5 \mathrm{ABCDEF}$ & $8.1 \pm 0.8^{\text {BCDEFG }}$ & $3.7 \pm 0.3 \mathrm{DE}$ & $25.8 \pm 0.9 \mathrm{ABCDE}$ \\
\hline Gala & $13.2 \pm 0.4 \mathrm{CDFF}$ & $8.1 \pm 0.2^{\text {BCDEFG }}$ & $4.5 \pm 0.4 \mathrm{ABC}$ & $25.8 \pm 1.0 \mathrm{ABCDE}$ \\
\hline La Paz & $14.6 \pm 0.7 \mathrm{ABCD}$ & $7.4 \pm 0.5^{\mathrm{DEFGH}}$ & $3.9 \pm 0.5^{\mathrm{CDE}}$ & $25.8 \pm 1.3 \mathrm{ABCDE}$ \\
\hline Pink Floyd & $12.6 \pm 0.3^{\mathrm{EF}}$ & $9.1 \pm 0.5^{\mathrm{AB}}$ & $4.2 \pm 0.4 \mathrm{BCDE}$ & $26.0 \pm 0.9 \mathrm{ABCDE}$ \\
\hline Harold & $12.9 \pm 0.7 \mathrm{DEF}$ & $8.8 \pm 0.1 \mathrm{ABC}$ & $4.4 \pm 0.3^{\mathrm{BC}}$ & $26.1 \pm 1.0 \mathrm{ABCD}$ \\
\hline Hatton & $14.2 \pm 1.0 \mathrm{ABCDE}$ & $7.5 \pm 1.2 \mathrm{DEFGH}$ & $4.4 \pm 0.3^{\mathrm{BC}}$ & $26.1 \pm 1.2 \mathrm{ABCD}$ \\
\hline Aifi Wuriti & $15.0 \pm 0.7 \mathrm{ABC}$ & $7.2 \pm 0.3 \mathrm{EFGH}$ & $4.1 \pm 0.3^{\mathrm{BCDE}}$ & $26.3 \pm 0.9 \mathrm{ABCD}$ \\
\hline Coulee & $13.6 \pm 1.2^{\mathrm{BCDEF}}$ & $8.1 \pm 0.2^{\mathrm{BCDEF}}$ & $4.5 \pm 0.3^{\mathrm{BC}}$ & $26.3 \pm 1.5 \mathrm{ABCD}$ \\
\hline Garnet & $14.0 \pm 1.0 \mathrm{ABCDEF}$ & $8.2 \pm 0.5^{\mathrm{BCDE}}$ & $4.2 \pm 0.4^{\mathrm{BCDE}}$ & $26.4 \pm 1.1 \mathrm{ABCD}$ \\
\hline Centa Pupil & $15.7 \pm 0.9^{\mathrm{A}}$ & $5.7 \pm 0.6^{\mathrm{I}}$ & $5.2 \pm 0.3^{\mathrm{A}}$ & $26.6 \pm 0.6 \mathrm{ABCD}$ \\
\hline Frontier & $13.9 \pm 0.3^{\mathrm{ABCDEF}}$ & $8.5 \pm 0.5^{\mathrm{BCD}}$ & $4.2 \pm 0.3^{\mathrm{BCDE}}$ & $26.7 \pm 0.4 \mathrm{ABCD}$ \\
\hline Rojo Chiquito & $15.4 \pm 1.4^{\mathrm{AB}}$ & $6.8 \pm 0.7 \mathrm{GHI}$ & $4.5 \pm 0.3^{\mathrm{BC}}$ & $26.7 \pm 1.0^{\mathrm{ABC}}$ \\
\hline Jackpot & $14.4 \pm 2.0 \mathrm{ABCDE}$ & $8.2 \pm 1.0^{\mathrm{BCDE}}$ & $4.4 \pm 0.4^{\mathrm{BC}}$ & $27.0 \pm 1.4^{\mathrm{AB}}$ \\
\hline Voyager & $13.6 \pm 0.8^{\mathrm{BCDEF}}$ & $9.8 \pm 0.7^{\mathrm{A}}$ & $4.0 \pm 0.3^{\mathrm{CDE}}$ & $27.4 \pm 0.8^{\mathrm{A}}$ \\
\hline MEAN & $13.9 \pm 1.1$ & $7.7 \pm 1.0$ & $4.2 \pm 0.4$ & $25.8 \pm 1.1$ \\
\hline$p$-value & 0.0001 & 0.021 & 0.007 & 0.008 \\
\hline
\end{tabular}

a Values are expressed as percent of dry weight; means $\pm \mathrm{SD} ;{ }^{\mathrm{b}}$ Means followed by different superscripted letters within a column are significantly different $(p<0.05)$ according to Tukey's mean separation test. Abbreviations: IDF: insoluble dietary fiber; SDF: soluble dietary fiber; OLIGO: total oligosaccharides (raffinose + stachyose + verbascose); TDF: total dietary fiber (IDF + SDF + OLIGO).

The average total dietary fiber for all common bean cultivars was $25.8 \%$, with a range of $24.1 \%$ to $27.4 \%$. The average IDF across cultivars was $13.9 \%$, while the range was $12.3 \%$ to $15.7 \%$. The average SDF for all cultivars was $7.7 \%$, with a range of $5.7 \%$ to $9.8 \%$. The average for total oligosaccharides was $4.2 \%$ with a range of $3.6 \%$ to $5.2 \%$. 


\subsection{Dry Pea}

Eleven dry pea entries were evaluated, which included eight varieties and three breeding lines from the USDA-ARS Grain Legume Genetics and Physiology Research Unit (Pullman, WA, USA). Selection of dry peas cultivars were from market classes commonly grown in North America. Seed was obtained from two locations (Washington State and Idaho), and within each location, seed was collected from two different field plots (replicates). Since the goal of this study was to determine concentrations of dietary fiber components and total dietary fiber in varieties of dry pea as they would be purchased and consumed, the four independent values for each variety were averaged and are reported in Table 3.

Table 3. Dietary fiber content of dry pea cultivars and breeding lines.

\begin{tabular}{|c|c|c|c|c|}
\hline Variety & $\operatorname{IDF}^{a, b}$ & $\operatorname{SDF}^{a, b}$ & OLIGO $^{a, b}$ & TDF $\mathbf{a}, \mathbf{b}$ \\
\hline HAMPTON & $14.6 \pm 1.4^{\mathrm{C}}$ & $3.6 \pm 0.7^{\mathrm{B}}$ & $4.1 \pm 0.9^{\mathrm{DE}}$ & $22.3 \pm 1.6^{C}$ \\
\hline PS03101445 & $14.2 \pm 2.2^{C}$ & $3.5 \pm 0.5^{\text {В }}$ & $5.2 \pm 0.4 \mathrm{AB}$ & $22.9 \pm 2.1^{C}$ \\
\hline PS071019971 & $15.5 \pm 1.4^{\mathrm{BC}}$ & $3.8 \pm 0.7^{\mathrm{B}}$ & $4.4 \pm 0.4 \mathrm{BCDE}$ & $23.8 \pm 1.7^{\mathrm{C}}$ \\
\hline DS ADMIRAL & $16.0 \pm 1.2^{\mathrm{BC}}$ & $3.5 \pm 0.4^{\mathrm{B}}$ & $4.3 \pm 0.4^{\mathrm{CDE}}$ & $23.8 \pm 1.6^{C}$ \\
\hline COLUMBIAN & $15.4 \pm 0.5^{\mathrm{BC}}$ & $3.3 \pm 0.6^{\mathrm{B}}$ & $5.4 \pm 0.3^{\mathrm{A}}$ & $24.1 \pm 0.6^{\mathrm{BC}}$ \\
\hline CAROUSEL & $15.0 \pm 1.4^{C}$ & $4.4 \pm 0.6^{\mathrm{AB}}$ & $4.8 \pm 0.7 \mathrm{ABCD}$ & $24.1 \pm 2.3^{B C}$ \\
\hline PS08101022 & $15.8 \pm 1.2^{\mathrm{BC}}$ & $3.7 \pm 1.5^{\text {В }}$ & $4.6 \pm 0.3 \mathrm{ABCDE}$ & $24.2 \pm 0.8^{\mathrm{BC}}$ \\
\hline ARAGORN & $14.8 \pm 1.7^{C}$ & $5.2 \pm 2.0^{\mathrm{A}}$ & $4.4 \pm 0.5 \mathrm{CDE}$ & $24.5 \pm 1.1^{\mathrm{BC}}$ \\
\hline SPECTER & $17.6 \pm 2.5^{\mathrm{AB}}$ & $4.1 \pm 0.5^{\mathrm{AB}}$ & $4.9 \pm 0.2 \mathrm{ABCD}$ & $26.7 \pm 3.0 \mathrm{AB}$ \\
\hline MELROSE & $19.8 \pm 2.9^{\mathrm{A}}$ & $3.9 \pm 0.7^{\mathrm{B}}$ & $4.0 \pm 0.2^{\mathrm{E}}$ & $27.6 \pm 2.5^{\mathrm{A}}$ \\
\hline GRANGER & $19.1 \pm 1.5^{\mathrm{A}}$ & $3.8 \pm 0.6^{\mathrm{B}}$ & $5.0 \pm 1.0 \mathrm{ABC}$ & $28.0 \pm 2.5^{\mathrm{A}}$ \\
\hline Overall Mean & $16.0 \pm 2.2$ & $3.9 \pm 1.0$ & $4.7 \pm 0.6$ & $24.6 \pm 2.4$ \\
\hline$p$-value & 0.004 & 0.079 & 0.026 & 0.006 \\
\hline
\end{tabular}

a Values are expressed as percent of dry weight; means $\pm \mathrm{SD}$; ${ }^{\mathrm{b}}$ Means followed by different superscripted letters within a column are significantly different $(p<0.05)$ according to Tukey's mean separation test. Abbreviations: IDF: insoluble dietary fiber; SDF: soluble dietary fiber; OLIGO: total oligosaccharides (raffinose + stachyose + verbascose); TDF: total dietary fiber (IDF + SDF + OLIGO).

The average total dietary fiber for all dry pea cultivars was $24.6 \%$, with a range of $22.3 \%$ to $28.0 \%$. The average for IDF across cultivars was $16.0 \%$, while the range was $14.2 \%$ to $19.8 \%$. The average SDF for all cultivars was $3.9 \%$, with a range between $3.3 \%$ and $5.2 \%$. The average for total oligosaccharides was $4.7 \%$, with a range between $4.1 \%$ and $5.4 \%$.

\subsection{Lentils}

Thirteen lentil entries were evaluated, which included 11 varieties and two breeding lines. The selection of lentils included cultivars from market classes commonly grown in North America. Seed was obtained from two locations in Washington State-Pullman and Dayton-and within each location, seed was collected from two different field plots (replicates). Since the goal of this study was to determine concentrations of dietary fiber components and total dietary fiber in cultivars of lentils as they would be purchased and consumed, the four independent values for each variety were averaged, and are reported in Table 4.

The average total dietary fiber for lentil cultivars was $20.1 \%$ with a range from $18.4 \%$ to $21.3 \%$. The average for IDF across cultivars was $13.6 \%$, while the range was $12.2 \%$ to $14.7 \%$. The average SDF for all cultivars was $3.2 \%$, with a range between $2.7 \%$ and $3.9 \%$. The average for total oligosaccharides was $3.3 \%$, with a range between $3.0 \%$ and $3.7 \%$. 
Table 4. Dietary fiber content of lentil cultivars and breeding lines.

\begin{tabular}{|c|c|c|c|c|}
\hline Variety & IDF $^{a, b}$ & SDF $a, b$ & OLIGO $^{a, b}$ & $\mathrm{TDF}^{\mathrm{a}, \mathrm{b}}$ \\
\hline Cedar & $12.2 \pm 1.0^{\mathrm{C}}$ & $3.1 \pm 0.0^{\mathrm{BCD}}$ & $3.0 \pm 0.1^{\mathrm{BC}}$ & $18.4 \pm 1.1^{\mathrm{F}}$ \\
\hline Morton & $13.7 \pm 0.8^{\mathrm{B}}$ & $2.7 \pm 0.1^{\mathrm{E}}$ & $3.0 \pm 0.0^{C}$ & $19.4 \pm 0.7^{\mathrm{E}}$ \\
\hline Pardina & $13.5 \pm 0.3^{\mathrm{B}}$ & $2.8 \pm 0.2^{\mathrm{DE}}$ & $3.2 \pm 0.1 \mathrm{ABC}$ & $19.5 \pm 0.1^{\mathrm{DE}}$ \\
\hline LC08600113P & $13.4 \pm 0.3^{B}$ & $3.0 \pm 0.1^{\mathrm{BCDE}}$ & $3.4 \pm 0.2^{\mathrm{ABC}}$ & $19.8 \pm 0.2^{\mathrm{CDE}}$ \\
\hline CDC Viceroy & $13.4 \pm 0.3^{\mathrm{B}}$ & $3.0 \pm 0.1 \mathrm{BCDE}$ & $3.4 \pm 0.3 \mathrm{ABC}$ & $19.8 \pm 0.5 \mathrm{CDE}$ \\
\hline Eston & $13.5 \pm 0.3^{B}$ & $3.0 \pm 0.1 \mathrm{BCDE}$ & $3.3 \pm 0.3^{\mathrm{ABC}}$ & $19.9 \pm 0.2^{\mathrm{CDE}}$ \\
\hline Richlea & $13.7 \pm 0.3^{\mathrm{B}}$ & $3.2 \pm 0.2^{\mathrm{BC}}$ & $3.1 \pm 0.2^{\mathrm{BC}}$ & $20.0 \pm 0.2^{C D}$ \\
\hline Avondale & $13.6 \pm 0.7^{\mathrm{B}}$ & $3.1 \pm 0.3^{\mathrm{BCD}}$ & $3.3 \pm 0.4^{\mathrm{ABC}}$ & $20.0 \pm 0.3^{C D}$ \\
\hline LC06601734L & $13.7 \pm 0.3^{B}$ & $3.3 \pm 0.1^{\mathrm{B}}$ & $3.3 \pm 0.3^{\mathrm{ABC}}$ & $20.3 \pm 0.3^{B C}$ \\
\hline Merrit & $13.5 \pm 0.8^{\mathrm{B}}$ & $3.3 \pm 0.1^{\mathrm{B}}$ & $3.5 \pm 0.2^{\mathrm{AB}}$ & $20.3 \pm 0.8^{B C}$ \\
\hline Crimson & $14.7 \pm 0.4^{\mathrm{A}}$ & $2.9 \pm 0.0^{\mathrm{CDE}}$ & $3.0 \pm 0.3^{C}$ & $20.6 \pm 0.7^{\mathrm{B}}$ \\
\hline Brewer & $13.8 \pm 0.6^{\mathrm{B}}$ & $3.3 \pm 0.1^{\mathrm{B}}$ & $3.7 \pm 0.2^{\mathrm{A}}$ & $20.8 \pm 0.4^{\mathrm{AB}}$ \\
\hline Pennell & $13.8 \pm 0.2^{\mathrm{B}}$ & $3.9 \pm 0.3^{\mathrm{A}}$ & $3.6 \pm 0.3^{\mathrm{A}}$ & $21.3 \pm 0.2^{\mathrm{A}}$ \\
\hline Overall Mean & $13.6 \pm 0.6$ & $3.2 \pm 0.3$ & $3.3 \pm 0.3$ & $20.1 \pm 0.7$ \\
\hline$p$-value & 0.0155 & $<0.0001$ & 0.1152 & $<0.0001$ \\
\hline
\end{tabular}

a Values are expressed as percent of dry weight; means $\pm \mathrm{SD} ;{ }^{\mathrm{b}}$ Means followed by different superscripted letters within a column are significantly different $(p<0.05)$ according to Tukey's mean separation test. Abbreviations: IDF: insoluble dietary fiber; SDF: soluble dietary fiber; OLIGO: total oligosaccharides (raffinose + stachyose + verbascose); TDF: total dietary fiber (IDF + SDF + OLIGO).

\subsection{Comparisons among Pulse Crops}

The data from Tables 1-4 were compiled and then subjected to further statistical analyses. Table 5 summarizes the mean values across the pulse crops for total dietary fiber and its principle components, insoluble and soluble fiber, and for total oligosaccharides (i.e., raffinose, stachyose, and verbascose).

Table 5. Dietary fiber content of pulse crops.

\begin{tabular}{|c|c|c|c|c|c|c|}
\hline Crop & IDF $^{a, b}$ & SDF $^{a, b}$ & OLIGO $^{a, b}$ & TDF $^{a, b}$ & TDF $^{c}$ g/Serving & $\mathrm{TDF}^{\mathrm{d}} \mathrm{g} / 100 \mathrm{kcal}$ \\
\hline Chickpea & $15.8 \pm 0.8^{\mathrm{A}}$ & $3.5 \pm 1.0^{\mathrm{A}, \mathrm{C}}$ & $2.5 \pm 0.7^{C}$ & $21.8 \pm 1.6^{\mathrm{B}}$ & 7.1 & 5.3 \\
\hline Common bean & $13.9 \pm 1.1^{\mathrm{B}}$ & $7.7 \pm 1.0^{\mathrm{B}}$ & $4.2 \pm 0.4^{\mathrm{A}}$ & $25.8 \pm 1.1^{\mathrm{A}}$ & 8.1 & 6.6 \\
\hline Dry pea & $16.2 \pm 1.9^{\mathrm{A}}$ & $3.9 \pm 0.5^{\mathrm{A}, \mathrm{C}}$ & $4.6 \pm 0.4^{\mathrm{A}}$ & $24.7 \pm 1.9^{\mathrm{A}}$ & 4.3 & 6.4 \\
\hline $\begin{array}{c}\text { Lentil } \\
p \text {-value }\end{array}$ & $\begin{array}{c}13.6 \pm 0.6^{\mathrm{B}} \\
<0.001\end{array}$ & $\begin{array}{c}3.1 \pm 0.3^{\mathrm{A}} \\
<0.001\end{array}$ & $\begin{array}{c}3.3 \pm 0.2^{B} \\
<0.001\end{array}$ & $\begin{array}{c}20.0 \pm 0.7^{C} \\
<0.001\end{array}$ & 6.0 & 5.2 \\
\hline
\end{tabular}

a Values are expressed as percent of dry weight; means with different superscripts within a column are significantly different $(p<0.05)$; ${ }^{\mathrm{b}}$ Means followed by different superscripted letters within a column are significantly different $(p<0.05)$ according to Tukey's mean separation test; ${ }^{c}$ Serving size is $\frac{1}{2}$ cup of cooked, drained pulse; ${ }^{\mathrm{d}}$ This is per $100 \mathrm{kcal}$ pulse crop as eaten. Abbreviations: IDF: insoluble dietary fiber; SDF: soluble dietary fiber; OLIGO: total oligosaccharides (raffinose + stachyose + verbascose); TDF: total dietary fiber (IDF + SDF + OLIGO)

All pulse crops in this study had a high content of total dietary fiber. Common bean and dry pea have the highest amounts, which were statistically different from chickpea and lentil; however, when these data were reported in terms of TDF per serving as consumed or per $100 \mathrm{kcal}$ as consumed, the relationships among pulse crops differed. Nonetheless, these differences are probably not clinically important, given the range in total dietary fiber across entries within each crop (Table 6), and the fact that identity preserved seed is currently not available to the consumer. Differences exist in dietary fiber components across pulse crops, and these are shown in Table 5. 
Table 6. Range of dietary fiber content (\%) among pulse crops.

\begin{tabular}{ccccc}
\hline Crop & IDF $^{\mathbf{a}}$ & SDF $^{\mathbf{a}}$ & OLIGO $^{\mathbf{a}}$ & TDF $^{\mathbf{a}}$ \\
\hline Chickpea & $14.4-17.1$ & $2.0-5.8$ & $1.0-3.5$ & $19.5-24.9$ \\
Common bean & $12.3-15.7$ & $5.8-9.8$ & $3.6-5.2$ & $24.1-27.4$ \\
Dry pea & $14.2-19.8$ & $3.3-5.3$ & $4.0-5.4$ & $22.3-28.0$ \\
Lentil & $12.3-14.7$ & $2.7-3.9$ & $3.0-3.7$ & $18.4-21.3$
\end{tabular}

a Values are expressed as percent of dry weight. Abbreviations: IDF: insoluble dietary fiber; SDF: soluble dietary fiber; OLIGO: total oligosaccharides (raffinose + stachyose + verbascose); TDF: total dietary fiber (IDF + SDF + OLIGO).

\section{Discussion}

Dietary fiber influences human health, due in part to its effects on physiological functions, which include: (1) decreased intestinal transit time and increased stool bulk; (2) increased fermentation by colonic microflora; (3) reduced low-density lipoprotein cholesterol; and (4) reduced postprandial blood glucose and/or insulin [18,19]. These physiological effects have also been reported to impact the risk for several chronic diseases, including obesity, type 2 diabetes, heart disease, and certain types of cancer $[20,21]$. However, inadequate dietary fiber intake is widespread, with less than $10 \%$ of all Americans meeting recommended intake levels-a fact that has prompted renewed efforts to close the fiber gap in our diet [2,3,19].

The dietary fiber gap has existed for decades, despite strong efforts to increase fiber intake through consumer education and the introduction of an array of fiber enriched food products [22,23]. During the same period of time, an epidemic rate of obesity has occurred, a situation that strongly argues that foods with a high content of dietary fiber per $100 \mathrm{kcal}$ portion should be emphasized in order to increase fiber intake without increasing caloric intake [24]. To this end, we have advanced evidence that the lack of pulse consumption in countries such as the United States is a significant contributor to the dietary fiber gap, since these crops provide two to three times more dietary fiber per $100 \mathrm{~g}$ edible portion than cereal grains, the type of foods frequently advertised as sources of dietary fiber [4]. The high protein and low fat content of pulses further strengthens the case for emphasizing their regular consumption in efforts to close the dietary fiber gap (Table 7).

Table 7. Dietary fiber, protein, and lipid content of pulse and cereal crops per $100 \mathrm{kcal}$ edible portion.

\begin{tabular}{|c|c|c|c|c|c|c|c|c|c|}
\hline Crop $^{a}$ & Bean $^{b}$ & Lentils $^{c}$ & Chickpeas $^{d}$ & Dry Pea ${ }^{e}$ & Wheat ${ }^{\mathrm{f}}$ & Rice $\mathrm{g}$ & Corn ${ }^{h}$ & Soy ${ }^{i}$ & Peanut ${ }^{j}$ \\
\hline Lipid g/100 kcal & 0.5 & 3.3 & 1.6 & 0.3 & 1.4 & 0.8 & 1.6 & 4.5 & 6.9 \\
\hline
\end{tabular}

${ }^{a}$ Values for food composition taken from USDA Nutrient Database for Standard reference: Release 28 ; ${ }^{\mathrm{b}}$ Bean, pinto, mature seeds, cooked, boiled. Database reference number: $16043 ;{ }^{\mathrm{c}}$ Lentils, mature seeds, cooked, boiled. Database reference number: $16070 ;{ }^{d}$ Chickpea, mature seeds, cooked, boiled. Database reference number: 16057; ${ }^{\mathrm{e}}$ Pea, green, cooked, boiled. Database reference number: $11305 ;{ }^{\mathrm{f}}$ Bread, whole wheat, commercially prepared. Database reference number: $18075{ }^{\mathrm{g}}{ }^{\mathrm{R}}$ Rice, brown, long grain, cooked. Database reference number: 20037; h Corn, sweet, yellow, cooked, boiled. Database reference number: 11168; i Soybeans, green, cooked, boiled. Database reference number: $11451{ }^{j}$ Peanuts, all types, cooked, boiled. Database reference number: 16088; ${ }^{\mathrm{k}}$ Values taken from Table 5.

Moreover, dietary surveys in both the United States and Canada demonstrate that pulse consumers in both countries exceed recommended levels of dietary fiber [8,9]. The magnitude of fiber intake in these pulse consumers is actually underestimated, since those studies used nutrient databases constructed before AOAC 2011.25 was published. Older methods reporting either crude fiber or acid detergent fiber and neutral detergent fiber values for pulse crops did not use enzymes in combination with chemicals for fiber extraction [25]. The crude fiber analysis isolates only a part of the insoluble fiber fraction, and greatly underestimates total dietary fiber content. Soluble fiber components are not measured in methods determining acid detergent fiber and neutral detergent fiber. The data in Tables 1-4 resolve this issue, providing the fiber content of chickpeas, common bean, dry 
peas, and lentils using AOAC 2011.25. To our knowledge, this is the first reported application of this method to these four pulse crops.

Of the seventeen pulses recognized by FAO, the four crops investigated in this study are the most widely consumed pulses globally [5]. Of these, common bean is consumed in the largest amount. However, consumers who decide to increase pulse consumption to improve their dietary fiber status will undoubtedly ask whether one pulse is better than the others. Table 5 was constructed with this question in mind. While there were significant differences in dietary fiber components among pulse crops, with common bean and dry peas being the highest in overall fiber content, we consider that the best answer to the consumers' question is to encourage consumption across pulse types, since it is recognized that consumption of a diverse array of dietary fiber components offers the approach most likely to facilitate tolerance of increasing levels of dietary fiber intake. Another common consumer question is whether specific components of dietary fiber should be emphasized (e.g., insoluble versus soluble dietary fiber). Historically, the answer to this question has varied, but the current consensus is that total fiber intake rather than consumption of a specific component is the most important consideration in terms of human health benefit $[3,19]$. With that in mind, a daily intake of $50 \mathrm{~g}$ dietary fiber, which was associated with a reduction in overall mortality in excess of $50 \%$ in the AARP cohort, might be considered a useful target intake for the health-oriented consumer [21].

A number of forces are driving a trend in consumer information and food labeling toward identity preservation of food sources, and in some cases, this extends to specific crop cultivars. Given that increasing dietary fiber intake is of paramount importance for human health and that there is no upper limit beyond which fiber intake is considered unsafe, Table 6 was constructed to summarize the amount of variation in dietary fiber components that exist among the cultivars of chickpea, common bean, dry pea and lentils that were evaluated [1]. The magnitude of these differences suggests that consideration should be given to cultivar labeling that underscores increased dietary fiber content in the absence of making any health claims. Choosing high versus low fiber cultivars of a particular pulse crop could boost total fiber intake by as much as 30\%, increasing the feasibility of reaching or exceeding recommended levels of consumption in the absence of an increase in caloric intake.

There are a number of issues related to pulse consumption and dietary fiber intake that merit additional comment. Pulse seeds must be cooked prior to consumption to inactivate lectins and to make them palatable. If seed is soaked before it is cooked, and the water is discarded, the oligosaccharide content can be lower, and thus total dietary fiber consumed reduced. While oligosaccharide consumption is associated with flatulence, oligosaccharides are also known to exert prebiotic effects, and thus their consumption is thought to be beneficial. It is also known that cooking can affect physical characteristics of dietary carbohydrate, and that is why AOAC 2011.25 was applied to cooked seed as reported herein [26].

\section{Conclusions}

To close the fiber gap, pulse crops should be emphasized in the diet, since they have two to three times more fiber per $100 \mathrm{~g}$ than other dietary staples. Substantial differences in fiber content among cultivars within a crop allows for additional gains in fiber intake without the need to change dietary habits, and suggests a rationale for cultivar-based food labeling.

Acknowledgments: This work was supported by USDA/NIFA Common Bean Coordinated Agricultural Project (BeanCAP), the American Pulse Association, the Colorado State University Agricultural Experiment Station, and PHS grant R01-CA172375 from the National Cancer Institute. The authors thank Dimas Echeverria, Barry Ogg, John McGinley, and Elizabeth Neil for their excellent technical assistance.

Author Contributions: H.T. and M.B. conceived and designed the experiments; Y.C. and H.T. performed the experiments; Y.C., H.T. and M.B. analyzed the data; R.M., G.V. and M.B. contributed materials; and Y.C. and H.T. wrote the paper.

Conflicts of Interest: The authors declare no conflict of interest. The founding sponsors had no role in the design of the study; in the collection, analyses, or interpretation of data; in the writing of the manuscript, and in the decision to publish the results. 


\section{Abbreviations}

The following abbreviations are used in this manuscript:

IDF Insoluble dietary fiber

SDF Soluble dietary fiber

OLIGO Oligossacharide

TDF Total dietary fiber

\section{References}

1. Otten, J.J.; Hellwig, J.P.; Meyers, L.D. Dietary Reference Intakes; Institute of Medicine of the National Academies: Washington, DC, USA, 2006.

2. Mobley, A.R.; Jones, J.M.; Rodriguez, J.; Slavin, J.; Zelman, K.M. Identifying practical solutions to meet America's fiber needs: proceedings from the Food \& Fiber Summit. Nutrients 2014, 6, 2540-2551. [PubMed]

3. Jones, J.M. CODEX-aligned dietary fiber definitions help to bridge the 'fiber gap'. Nutr. J. 2014, 13, 34. [CrossRef] [PubMed]

4. Thompson, H.J.; Brick, M.A. Perspective: Closing the dietary fiber gap: An ancient solution for a 21st century problem. Adv. Nutr. 2016, 7, 623-626. [CrossRef] [PubMed]

5. Food and Agriculture Organization. Definition and Classification of Commodities: Pulses and Derived Products. Available online: http:/ /www.fao.org/es/faodef/fdef04e.htm (accessed on 18 October 2015).

6. Gepts, P.; Famula, T. Biodiversity in Agriculture: Domestication, Evolution, and Sustainability; Cambridge University Press: Cambridge, UK, 2012.

7. Bressani, R. Research needs to up-grade the nutritional quality of common beans (Phaseolus vulgaris). Plant Foods Hum. Nutr. 1983, 32, 101-110. [CrossRef]

8. Mitchell, D.C.; Lawrence, F.R.; Hartman, T.J.; Curran, J.M. Consumption of dry beans, peas, and lentils could improve diet quality in the US population. J. Am. Diet. Assoc. 2009, 109, 909-913. [CrossRef] [PubMed]

9. Mudryj, A.N.; Yu, N.; Hartman, T.J.; Mitchell, D.C.; Lawrence, F.R.; Aukema, H.M. Pulse consumption in Canadian adults influences nutrient intakes. Br. J. Nutr. 2012, 108, S27-S36. [CrossRef] [PubMed]

10. Hipsley, E.H. Dietary "fibre" and pregnancy toxaemia. Br. Med. J. 1953, 2, 420-422. [CrossRef] [PubMed]

11. Burkitt, D.P.; Walker, A.R.; Painter, N.S. Effect of dietary fibre on stools and the transit-times, and its role in the causation of disease. Lancet 1972, 2, 1408-1412. [CrossRef]

12. Trowell, H. Definition of dietary fiber and hypotheses that it is a protective factor in certain diseases. Am. J. Clin. Nutr. 1976, 29, 417-427. [PubMed]

13. Trowell, H.C.; Burkitt, D.P. The development of the concept of dietary fibre. Mol. Aspects Med. 1987, 9, 7-15. [CrossRef]

14. Alimentarius CODEX (CODEX) Guidelines On Nutrition Labeling CAC/GL 2-1985. Available online: http:/ /www.fao.org/ag/humannutrition/33309-01d4d1dd1abc825f0582d9e5a2eda4a74.pdf (accessed on 15 October 2016).

15. McCleary, B.V.; DeVries, J.W.; Rader, J.I.; Cohen, G.; Prosky, L.; Mugford, D.C.; Champ, M.; Okuma, K. Determination of total dietary fiber (CODEX definition) by enzymatic-gravimetric method and liquid chromatography: collaborative study. J. AOAC Int. 2010, 93, 221-233. [CrossRef] [PubMed]

16. McCleary, B.V.; DeVries, J.W.; Rader, J.I.; Cohen, G.; Prosky, L.; Mugford, D.C.; Okuma, K. Determination of insoluble, soluble, and total dietary fiber (CODEX definition) by enzymatic-gravimetric method and liquid chromatography: collaborative study. J. AOAC Int. 2012, 95, 824-844. [CrossRef] [PubMed]

17. Kleintop, A.E.; Echeverria, D.; Brick, L.A.; Thompson, H.J.; Brick, M.A. Adaptation of the AOAC 2011.25 integrated total dietary fiber assay to determine the dietary fiber and oligosaccharide content of dry edible beans. J. Agric. Food Chem. 2013, 61, 9719-9726. [CrossRef] [PubMed]

18. Cho, S.S.; Nelson, A. Dietary Fiber and Health; CRC Press: Boca Raton, FL, USA, 2012; p. 557.

19. Jones, J.M. Dietary fiber future directions: integrating new definitions and findings to inform nutrition research and communication. Adv. Nutr. 2013, 4, 8-15. [CrossRef] [PubMed]

20. Cho, S.S.; Qi, L.; Fahey, G.C., Jr.; Klurfeld, D.M. Consumption of cereal fiber, mixtures of whole grains and bran, and whole grains and risk reduction in type 2 diabetes, obesity, and cardiovascular disease. Am. J. Clin. Nutr. 2013, 98, 594-619. [CrossRef] [PubMed] 
21. Park, Y.; Subar, A.F.; Hollenbeck, A.; Schatzkin, A. Dietary fiber intake and mortality in the NIH-AARP diet and health study. Arch. Intern. Med. 2011, 171, 1061-1068. [CrossRef] [PubMed]

22. McRorie, J.W., Jr. Evidence-based approach to fiber supplements and clinically meaningful health benefits, part 1: What to look for and how to recommend an effective fiber therapy. Nutr. Today 2015, 50, 82-89. [CrossRef] [PubMed]

23. McRorie, J.W., Jr. Evidence-based approach to fiber supplements and clinically meaningful health benefits, part 2: What to look for and how to recommend an effective fiber therapy. Nutr. Today 2015, 50, 90-97. [CrossRef] [PubMed]

24. International Organization for Migration (IOM). Accelerating Progress in Obesity Prevention: Solving the Weight of the Nation. Available online: www.iom.edu/Reports/2012/Accelerating-Progressin-ObesityPrevention.aspx (accessed on 7 July 2016).

25. Tosh, S.M.; Yada, S. Dietary fibres in pulse seeds and fractions: Characterization, functional attributes, and applications. Food Res. Int. 2010, 43, 450-460. [CrossRef]

26. Brummer, Y.; Kaviani, M.; Tosh, S.M. Structural and functional characteristics of dietary fibre in beans, lentils, peas and chickpeas. Food Res. Int. 2015, 67, 117-125. [CrossRef]

(C) 2016 by the authors; licensee MDPI, Basel, Switzerland. This article is an open access article distributed under the terms and conditions of the Creative Commons Attribution (CC-BY) license (http://creativecommons.org/licenses/by/4.0/). 